\title{
Comenius (1592-1670) et le plurilinguisme
}

Jean Antoine Caravolas

\section{(2) OpenEdition \\ 12 Journals}

Édition électronique

URL : https://journals.openedition.org/dhfles/826

DOI : $10.4000 /$ dhfles.826

ISSN : 2221-4038

Éditeur

Société Internationale pour l'Histoire du Français Langue Étrangère ou Seconde

\section{Édition imprimée}

Date de publication : 1 décembre 2009

Pagination : 25-39

ISSN : 0992-7654

\section{Référence électronique}

Jean Antoine Caravolas, "Comenius (1592-1670) et le plurilinguisme », Documents pour I'histoire du français langue étrangère ou seconde [En ligne], 43 | 2009, mis en ligne le 16 janvier 2011, consulté le 27 mai 2021. URL : http://journals.openedition.org/dhfles/826 ; DOI : https://doi.org/10.4000/dhfles.826

Ce document a été généré automatiquement le 27 mai 2021.

(c) SIHFLES 


\title{
Comenius (1592-1670) et le plurilinguisme
}

\author{
Jean Antoine Caravolas
}

\section{Introduction}

1 Dans l'Appel de communicationsde la SIHFLES pour ce colloque on lit entre autres : « La didactique du plurilinguisme s'impose aujourd'hui comme une approche incontournable dans l'enseignement/apprentissage des langues en Europe ». Cela pourrait faire croire que la nécessité d'une formation massive de jeunes multilingues et par conséquent le besoin d'une didactique du plurilinguisme n'apparaît qu'après 1957 avecla création de l'Union européenne ${ }^{1}$,ou après 1990, avec l'émergencede la mondialisation $^{2}$ et des mouvements antimondialistes, ce qui serait faux. On oublie souvent que la didactique des langues a un très long passé. Cet oubli n'est pas toujours innocent. À ma connaissance, le premier à s'occuper de plurilinguisme et de didactique $\mathrm{du}$ plurilinguisme est Jan Amos Comenius (en tchèque Komenský). Comme vous connaissez tous qui il était et la place qu'il occupe dans l'histoire des idées ${ }^{3}$, je passe tout de suite à mon sujet :Comenius et le plurilinguisme.

\section{Comenius théoricien du multilinguisme}

2 L'intérêt de Comenius pour le multilinguisme commence vers 1628, quand il écrivaitDidaktika (la version originelle, en langue tchèque, de la Grande didactique). Il avait alors trente-six ans. Cet intérêt ne le quitta plus jamais et celapour plusieurs raisons : au XVII ${ }^{e}$ siècle le monde continue à s'élargir (découverte de l'Australie en 1605, par le Hollandais Willem Janszoon); l'exploration de l'Afrique, de l'Asie et de l'Amérique se poursuit; la colonisation des territoires récemment explorés s'intensifie; les contacts internationaux se multiplient mais les barrières linguistiques restent, ce qui entrave la communication entre les individus et les peuples et rend 
l'évangélisation des païens dans le vieux et le nouveau monde, vivement poursuivie par les catholiques aussi bien que par les protestants, très difficile.

Outre les raisons évoquées, Comenius avait une raison personnelle de s'intéresser au multilinguisme. Il était convaincu d'être chargé par le Ciel d'une mission de la plus grande importance pour l'humanité : préparer, avec quelques autres élus, l'avènement $\mathrm{du}$ règne millénaire $\mathrm{du}$ Christ, dont le retour sur terre était prévu pour très bientôt ${ }^{4}$, peut-être déjà en 1666, 1672 ou 1673.

Instaurer le royaume du Christ sur terre, signifiait pour Comenius transformer la vie quotidienne des hommes de telle manière qu'elle ressemble le plus possible à celle qui attendait les justes après la mort. Le succès de sa tâche dépendait en grande partie de la rapidité avec laquelle l'heureuse nouvelle (l'imminence du millénium) serait répandue, expliquée et crue par les hommes de tous les pays et de toutes les origines. Il fallait pour cela trouver vite une manière efficace de les informer.

5 Comenius pensait que le moyen le plus sûr et le plus rapide d'y parvenir serait d'assurer, sans tarder, l'éducation formelle et méthodique de tous les enfants de la terre, et cela à l'école. L'éducation des jeunes se fait en règle générale par l'exemple et la parole. Mais depuis Babel les hommes ne parlent plus tous la même langue. Comment contourner ce formidable obstacle ? En quelle(s) langue(s) dispenser l'enseignement pour que tous les intéressés comprennent et apprennent tout, solidement et incessamment? Comenius entrevoit quatre réponses possibles à cette question : l'enseignement unilingue (monoglossie), l'enseignement bilingue (diglossie), l'enseignement multilingue (polyglossie) et l'enseignement en toutes les langues connues (panglossie).

6 1. Enseignement unilingue. L'imposition de la monoglossie serait, à son avis, la solution la plus naturelle: les premiers hommes parlaient tous la même langue, la langue "adamique ». Il suffirait donc de la réintroduire. Hélas, il y a longtemps qu'elle a été oubliée. Il est vrai que l'érudit anglais John Dee (1527-1608 ?) s'enorgueillit dans son journal (voir Peterson 2003) d'avoir réussi, pendant des séances de magie, à communiquer avec les anges, notamment avec l'archange Raphäl et que ce dernier lui révéla, entre autres, un livre saint (les prophéties perdues d'Énoch), écrit dans la langue adamique et un alphabet magique. Dee donne même des exemples de cette écriture inconnue ainsi que deux lignes du texte écrit en adamique. Le reste du document révélé cependant, il l'écrit en caractères latins, n'ayant jamais apprit à se servir couramment de la langue des anges (Findell $2007: 7-22$ ).

7 2. Enseignement bilingue. La deuxième solution serait d'exiger de tous la maitrise d'une langue seconde commune. Le bilinguisme (diglossie) cependant se heurte à de nombreux obstacles: outre les jalousies nationales, aucune langue naturelle n'est parfaite et toutes sont plus ou moins difficiles à apprendre, à cause des irrégularités qu'elles présentent. On pourrait certes créer une langue artificielle, philosophiqueet parfaite ${ }^{5}$ mais cela demanderait beaucoup de temps et d'efforts et le temps, comme il a été déjà dit, pressait.

8 3. Enseignement en toutes les langues. La troisième solution serait de décréter le panglottisme (la panglossie). Mais cela est pratiquement impossible et aussi inutile. Impossible à cause du nombre de langues utilisées à travers le monde ${ }^{6}$, inutile parce que personne n'aura jamais besoin dans sa vie d'utiliser toutes les langues naturelles; 
puis, apprendre des langues n'est pas facile ; enfin, les hommes doivent consacrer leur temps, surtout quand ils sont jeunes, à étudier les choses pas à apprendre des mots.

4. Tout compte fait, la solution la plus raisonnable serait, lui semble-t-il, d'imposer l'enseignement multilingue. L'usage de plusieurs langues (polyglossie ${ }^{7}$ ou plurilinguisme ${ }^{8}$ ) est après la confusion de Babel devenu une nécessité : «Au lieu d'une seule langue, il nous faut désormais en apprendre plusieurs si nous voulons nous instruire, échanger des idées ou lire les auteurs disparus" (Comenius 1992: 70). La polyglossie est non seulement une pratique ancienne et éprouvée par le temps, elle est aussi une pratique louable: "L'exemple de Caton, de Mithridate et des apôtres montre à l'évidence que c'est une gloire de savoir plusieurs langues » (Comenius $2005: 379$ ).

\section{La conception coménienne du multilinguisme}

La conception du multilinguisme de Comeniusest liée à sa théorie de l'éducation. Sa théorie de l'éducation est exposée notamment dans la Grande didactique (1657/1992), La Toute nouvelle Méthode des langues (1648/2005) et dans le quatrième et le cinquième livres de la Consultation universelle pour la réforme des affaires humaines (1966) (la Pampaedie et la Panglottie). Tous ces ouvrages s'appuient sur sa théorie de la pansophie9

11 Comenius définit sa pansophie comme une philosophie chrétienne fondée sur la synthèse " harmonieuse » des données fournies par les sens, la raison et les révélations (les Saintes Écritures). Il espérait la résumer bientôt dans un ouvrage qui exposerait " avec art et méthode tout ce qu'il faut savoir et croire, faire et espérer pour parvenir au bonheur dans cette vie et dans la vie future " (Continuatio admonitionis fraternae (1659:\$47, à paraître en français).

12 Comenius croyait que l'acquisition de la pansophie était indispensable à tous et que tous pouvaient et devaient devenir pansophistes. Ils devaient, parce qu'ils ont été créés dans le but de devenir des images fidèles de leur Créateur. Ils pouvaient, s'ils étaient bien préparés à l'école. « Pour former la jeunesse il faut ouvrir des écoles ${ }^{10}$ » (Comenius 1992 : 77). Le philosophe tchèque Patočka, appelle la pansophie coménienne, «utopie des utopies » $(1998: 276)$.

Selon Comenius, l'éducation dispensée par les écoles devait être universelle : « Tous les enfants, nobles ou roturiers, riches ou pauvres, garçons ou filles de toutes les villes, cités, villages et hameaux doivent être admis dans les écoles; voilà ce dont il faut se convaincre » (Comenius 1992: 81). Il y a plusieurs raisons pour lesquelles l'éducation doit être universelle. La principale est que l'homme a pour fin de devenir une « fidèle image du créateur. Ce sont donc tous les enfants qu'il faut éduquer au savoir, à la vertu et à la religion. Il faut les rendre aptes à la vie présente et dignes de la vie future " (ibid.). Ensuite, «[...] si nous développons chez certains seulement l'intelligence et la culture, les autres en étant exclus, nous sommes injustes envers des êtres qui ont la même nature fondamentale, mais aussi contre Dieu qui veut être reconnu et loué par tous ceux en qui il a imprimé son image». Puis, "nous ne savons pas à quelles occupations nous destine la providence divine». Enfin, "plus un enfant est d'esprit faible et de nature stérile plus il exige de secours pour sortir de sa faiblesse et de sa stupidité » (ibid.).

Comenius était persuadé que l'éducation universelle était non seulement nécessaire mais aussi possible, car « l'homme naît apte à acquérir la connaissance des choses ». Il 
est doté d'une « âme raisonnable », d'organes "grâce auxquels elle atteint ce qui lui est extérieur » et de dispositions innées. "Ainsi [...] il n'est rien que l'homme ne puisse comprendre par les sens ou la raison» (ibid. : 60-61) ${ }^{11}$. Demander que l'éducation soit universelle était, hier comme aujourd'hui, une revendication révolutionnaire. Le cardinal de Richelieu, contemporain de Comenius, mais pas seulement lui, le comprit tout de suite. Dans son Testament politique (1985 : 117), il écrit :

Comme la connaissance des lettres est tout à fait necessaire en une Republique, il est certain qu'elles ne doivent pas estre indifferemment enseignées à tout le monde. Ainsi qu'un corps qui auroit des yeux en toutes ses parties, seroit monstrueux : de mesme un Etat le seroit-il, si tous ses Sujets etoient sçavans; on y verroit aussi peu d'obeissance que 1'orgueil et la presomption y seroient ordinaires.

Mais il ne suffit pas que l'éducation soit universelle, elle doit être aussi pansophique :

[...] l'ensemble des hommes a intérêt à apprendre, en spectateur mais aussi en acteur, ce qui est et advient, les fondements, les causes et les effets de tout ce vaste monde. Il faut donc parvenir à une organisation de l'école telle que nul par la suite ne rencontre rien qui lui soit inconnu et dont il ne puisse user utilement tout en évitant les erreurs (Comenius $1992: 85$ ).

Que signifie éducation pansophique? «Cela ne signifie pas que tous doivent acquérir la connaissance de tous les arts et de toutes les sciences, encore moins de leur connaissance complète et exacte. La tâche serait inutile par nature et impossible vu la brièveté de la vie » (ibid.).

Revendiquer une éducation universelle et pansophique est une demande subversive. En effet, imaginez un monde où «nul [...] ne rencontre rien qui lui soit inconnu " (ibid. : 81). A quoi lui serviraient alors les rois, les maîtres, les juges et les prêtres? Les puissants du monde s'en épouvantaient mais Comenius s'en réjouissait: "Si on m'objecte : «Où allons-nous si les ouvriers, les paysans, les portefaix, et pour finir les faibles femmes se mettent à l'instruction?", je répondrai qu'une instruction de la jeunesse en général, lorsqu'elle suivra la bonne voie, permettra à chacun de penser, de choisir convenablement et de bien agir » (ibid : 83)

\section{Pour une éducation multilingue}

L'éducation universelle pansophique est inconcevable sans une éducation multilingue,aucune langue ne contenant toute la sagesse humaine et divine. La sagessese cache dans la tradition orale et écrite, pas d'une mais de toutes les nations, de tous les peuples, de toutes les tribus. Il est donc évident que pour acquérir la sagesse il faut connaître, outre sa langue maternelle, les langues étrangères dont on a ou on aura un jour besoin.

\section{Selon Comenius :}

Sont nécessaires la langue maternelle pour traiter des affaires domestiques et les langues des pays voisins pour entrer en relation avec eux [...]. Puis vient le latin, langue savante qui permet de lire les ouvrages scientifiques. Enfin les philosophes et les médecins apprendront le grec et l'arabe, les théologiens le grec et l'hébreu (ibid. : 191).

Lors de l'apprentissage d'une langue il est indispensable de ne jamais oublier que la langue n'est qu'un outil et que l'important ne sont pas les mots qu'elle possède mais les pensées et les connaissances qu'elle transmet. Apprendre une langue est difficile. Appendre plusieurs langues est très difficile et, ajoute Comenius, dangereux. Pour cela 
il conseille à tous de na pas tenter « d'apprendre toutes les langues à la perfection, mais seulement dans la limite du nécessaire $»^{12}$ et de limiter le nombre de langues à apprendre ${ }^{13}$.

Toutefois, je crois de mon devoir de rappeler l'importance d'user de modération dans l'étude des langues, quelque facilitée qu'elle soit, pour éviter qu'on n'y mette trop de zèle. Pourquoi ? Pour la raison que, quelle que soit la rapidité avec laquelle on étudie les langues on ne peut toutefois pas procéder de manière à sacrifier le temps qui doit être accordé à l'étude de la réalité, parce qu'alors, les possibilités mêmes de l'intelligence et de la mémoire seraient débordées au point où il n'y aurait peut-être plus de place pour la réalité. En effet, le soin consacré à la maîtrise de la réalité doit être proportionnel à l'usage qu'on en fera. Il est certain que plus on connait de langues, plus il y a raison de craindre de jeter de la confusion dans l'intelligence. C'est ainsi qu'on sait que des cerveaux sont tombés dans une sorte d'hébétude et de stupidité, pour s'être tenus trop occupés à l'étude de nombreuses langues (Comenius $2005: 384$ ).

Il ajoute :

Si quelqu'un m'oppose l'exemple de Mithridate, roi du Pont, dont Pline raconte qu'il aurait parlé les vingt deux langues des peuples sur lesquels il régnait, je répondrai qu'en effet Pline dit cela mais que c'est peu probable, car il raconte cela sur la foi d'un autre. Cela va d'ailleurs au-delà de la croyance à l'effet qu'il y aurait eu autant de langues différentes dans le si petit nombre de pays sur lesquels s'est étendue la royauté de ce roi (ibid.).

\section{Comenius théoricien de la didactique du multilinguisme}

Comenius est également le premier auteur à proposer une esquisse de théorie de la didactique du multilinguisme. Il soutenait que les difficultés d'apprentissage des langues diminueraient considérablement si :

1.- les autorités compétentes adoptaient son projet d'éducation universelle, décrit dans la Grande Didactique;

2.- les enseignants acceptaient sa méthode universelle d'enseignement des langues, expliquée dans la Methodus Linguarum Novissima et dans la Panglottie ;

3.- et si les élèves apprenaient les langues dans ses manuels universels (Vestibulum, Janua, Atrium = VIA).

$\mathrm{Si}$, en outre, ces trois livres étaient traduits dans toutes les langues nationales et chaque mot de chaque langue vivante était placé en parallèle du mot latin du texte original, l'étude des langues étrangères deviendrait une affaire si non facile, certainement moins pénible qu'elle est à présent. À ceux qui néanmoins souhaitaient devenir pansophistes polyglottes tout de suite et vite, Comenius conseillait de suivre les sept règles suivantes :

1.- «Un temps déterminé sera consacré à l'étude de chaque langue ». Cependant, l'étude de la langue maternelle, base de l'apprentissage des "choses» et de toutes les langues (mortes ou vivantes), exige plus d'années que les autres. Ainsi, si on consacre deux ans à l'étude du latin on consacrera dix ans à l'étude de la langue vernaculaire.

2.- "Toute langue doit être apprise par l'usage plus que par les règles». Cela signifie en la faisant écouter, parler, lire et copier, le plus souvent possible.

3.- "Les règles cependant doivent venir pour éclairer et confirmer l'usage ». Ce principe vaut non seulement pour les règles savantes mais aussi pour l'étude des langues vivantes, 
car les plus cultivées d'entre elles (l'italien, le français, l'allemand, etc.) ont « des règles explicitement formulées".

4.- «Les règles de la langue sont grammaticales et non philosophiques ». L'étude des subtilités linguistiques sera réservée aux élèves de la classe de philosophie. Les autres se satisferont d'apprendre « ce que l'on fait et comment on le fait ».

5.- "L'étude de la langue nouvelle se règlera sur la norme de la langue déjà connue, en n'insistant que sur les différences ». Il est inutile d'enseigner, par exemple, les définitions du nom, du verbe, des cas ou des temps, etc. du grec. Les élèves les connaissent de l'étude du latin. On ne leur enseignera donc que ce qui en grec diffère du latin qu'ils ont déjà étudié.

6.- «Les exercices dans la langue nouvelle doivent traiter de matières connues ». De cette façon l'attention des élèves se portera seulement sur les mots, les choses que les mots désignent leur étant déjà connues de leur étude de la langue maternelle ou d'une langue étrangère.

7.- "Toutes les langues peuvent donc s'apprendre par une même et unique méthode ». Cette méthode se résume ainsi: exposition à l'usage en fournissant des exemples présentation de la règle qui l'applique à l'usage donné - beaucoup d'exercices d'application de la règle sur des matières connues, familières. On apprendra donc la nouvelle langue en imitant d'abord les exemples, ensuite en appliquant les règles et à la fin en faisant beaucoup d'exercices. C'est la règle d'or de la didactique coménienne (Comenius 1992 : 193-196).

En 1657 cependant, suite à la publication de Lux in tenebris ${ }^{14}$ et aux violentes polémiques que cet ouvrage provoqua, la réputation de Comenius souffre de manière irréparable. Son prestige baisse rapidement, l'auteur est ridiculisé, l'intérêt pour son œuvre s'estompe et après sa mort, en 1670, disparaît presque complètement. Même Opera Didactica Omnia ${ }^{15}$ (1657) ne sont épargnés. Le temps de Comenius avait passé. C'était la fin du grand siècle. Pierre Bayle, un des premiers précurseurs du siècle des Lumières, écrivait à propos des ODOdans son Dictionnaire historique et critique, qui allait devenir une des principales sources d'information des encyclopédistes : La république des lettres n'a tiré aucun profit [...] rien de praticable utilement dans les idées de cet auteur " (ibid.: 261). Mais Bayle se trompait et avec lui tous ceux, Français ou autres, qui partageaient son opinion. Les ODOcontiennent plusieurs chefs-d'œuvre, dont Didactica Magna, Methodus linguarum novissima et Schola materna. La Grande didactique est aujourd'hui universellement considérée comme un texte fondamental des sciences de l'éducation. La toute dernière méthode des langues, beaucoup moins connue, posa les fondements théoriques de la pédagogie des langues en tant que discipline autonome. Quant à L'école du giron maternel, ignoré complètement par les Français, est à juste titre célébré comme un des cent textes les plus importants publiés au cours du deuxième millénaire. C'est l'ouvrage qui découvrit au monde ce qu'est l'enfance (Life magazine, 1998 numéro spécial pour le nouveau millénaire) et cela bien avant J.-J. Rousseau, Froebel ou Pestalozzi.

Le rejet de Comenius par Bayle, Diderot et autres Français célèbres, lus, écoutés et admirés causa un énorme préjudice à Comenius et à la France. Pendant plus de deux cents ans, son œuvre, présentée comme le délire d'un mystique, un excentrique, un farfelu, sans rapport avec la vraie science, était ridiculisée, discréditée et dévalorisée et pour cela ni traduite, ni publiée ni étudiée. L'Allemagne, par contre, où les piétistes ${ }^{16}$ gardaient vivant l'héritage de Comenius et, plus tard, Herder ${ }^{17}$ et les néo-humanistes ${ }^{18}$ 
se plaça, dès le $18^{\mathrm{e}}$ siècle à la tête du renouveau pédagogique en Europe (voir Durkheim 1938/1990 : 327-333).

Il faudra attendre la fin de la Deuxième guerre mondiale et surtout l'année 1992, pour que les écrits de l'évêque de l'Union des Frères soient redécouverts et appréciés. Aujourd'hui les idées de Comenius sur l'éducation des jeunes et sur la pédagogie des langues, les deux domaines où il excellait, sont adoptées par l'Union européenne et adaptées aux conditions actuelles. Le principe de l'éducation universelle, de l'instruction d'abord dans la langue maternelle, de l'enseignement méthodique (non improvisé), de l'enseignement multilingue, de l'enseignement adapté au niveau intellectuel des élèves, de l'apprentissage actif, de l'apprentissage dans la joie (« schola ludus »), sont généralement acceptés, même s'ils ne sont pas partout appliqués.

Aussi, l'Organisation des Nations Unies pour l'Éducation, la Science et la Culture reconnaissant que la conception coménienne de l'éducation anticipe les idéaux et les objectifs de l'UNESCO, a créé, en 1992, à l'occasion du $400^{\mathrm{e}}$ anniversaire de la naissance de Comenius, conjointement avec le Ministère de l'éducation nationale de la République tchèque, la médaille Comenius. Elle est décernée aux pédagogues de tous les pays du monde qui font des contributions exceptionnelles dans le domaine de la recherche et de l'innovation dans l'enseignement supérieur.

Ainsi, peu à peu, se réalise la prophétie de Leibniz qui, en 1671, écrivait : « Tempus, erit, Comeni, quo te turba bonorum Factaque tua spesque tuas vota quoque ipsa colet » (Le temps viendra Comenius, où la foule des hommes de bien t'honorera, ainsi que tes œuvres, tes espérances et tes vœux).

\section{BIBLIOGRAPHIE}

BAYLE, Pierre. 1969. Dictionnaire historique et critique. Réimpression de l'éd.de 1820-1824, $16 \mathrm{v}$. Genève : Slatkine Reprints.

CARAVOLAS, Jean Antoine. 2000. Histoire de la didactique des langues au siècle des Lumières. Précis et anthologie thématique. Montréal, Tübingen : Les Presses de l'université de Montréal - Gunter Narr Verlag.

COMENIUS, Jan Amos. 1657/1631. Janua linguarum Reserata, in Opera Didactica Omnia (ODO)I.1 : 250-301. Amsterdam [Édition en fac-similé], Prague : CSAV.

------1657/1633. Schola infantiae (L'École du giron maternel)in ODO I.-1 : 198-249. Amsterdam [Édition en fac-similé], Prague : CSAV.

------1657/1633.Vestibulum in ODO I-1 :303-217. Amsterdam [Édition en fac-similé], Prague : CSAV.

----1657/1637. PansophiaeProdromus in ODO I.-1 : 404-454.Amsterdam [Édition en fac-similé], Prague : CSAV.

-----1957 [1657]. Opera Didactica Omnia (ODO), I pars 1-2 ; II, pars 3-4. Amsterdam [Édition en facsimilé], Prague : CSAV. 
-----1657. Lux in tenebris. Amsterdam.

------1657. In Latinitatis Atrium in ODO II, 1 :540-712. Amsterdam [Édition en fac-similé], Prague : CSAV.

------1657. Didactica Magna in ODO I-1 : 3-192. Amsterdam [Édition en fac-similé], Prague : CSAV.

------1966. De rerum humanarum emandatione consultatio catholica (Consultation Universelle pour la réforme des affaires humaines). 2 v. Pragae : Sumptibus Academiae Scientiarum Bohemoslovacae, Academia.

-----1989 [1648]. Novissima Linguarum Methodus, in ODO 15/II. pp. 92-402. Praha : Academia.

------1992. La Grande Didactique ou l'art universel de tout enseigner à tous. Paris : Klincksieck.

------2005. La toute nouvelle méthode des langues. Methodus Linguarum Novissima. Traduit du latin par Honoré Jean avec l'aide de Gilles Bibeau, Jean Antoine Caravolas et Claire Le Brun-Gouanvic. Genève : Droz.

------ (à paraître). Jan Amos Comenius. Suite de l'admonition fraternelle à Maresius. (Continuatio admonitionis fraternae). Traduit du latin par Claire Le Brun-Gouanvic.

DURKHEIM, Émile. 1938/1990. L'évolution pédagogique en France. Paris : PUF.

FINDELL, Martin. 2007. «The 'Book of Enoch', the Angelic Alphabet and the 'Real Cabbala' " in The Angelic Conferences of John Dee (1527-1608/9), Henry Sweet Society for the History of Linguistic Ideas BULLETIN $48: 7-22$.

McLUHAN, Marshall. 1962. The Gutenberg Galaxy: The Making of Typographic Man. Toronto : University of Toronto Press.

PATOČKA, Jan. 1998. Sebrané spisy Jana Patočky, sv.10 Komeniologické studie II. Utopie a soustavalidských cílů u Komenského. Praha : OIKOYMENH, 271-282.

PETERSON, Joseph H. (ed.). 2003. John Dee's Five Books of Mystery.York Beach, ME : Weiser.

RICHELIEU, Armand Jean du Plessis, Cardinal duc de. 1689. Testament politique. À Amsterdam : chez Henry Desbordes, [...] reprographie de l'Université de Cannes, Centre de Philosophie politique et juridique, 1985.

The LIFE Millennium.1998. The 100 most important events \& people of the past 1000 years. A Pictorial History of the world and the discoveries that changed it. Editor Robert Friedman. New York: Published by LIFE BOOKS Time Inc.

\section{NOTES}

1. La Communauté Économique Européenne a été instaurée le 25 mars 1957 par la France, la République fédérale allemande, l'Italie, la Belgique, les Pays-Bas et le Luxembourg.

2. On parle de mondialisation à partir de 1962, quand McLuhan publie The Gutenberg Galaxy : The Making of Typographic Man, où il explique comment à l'ère électronique le monde se transforme en un « village global ».

3. Les lecteurs intéressés trouveront facilement les informations qu'ils désirent sur sa vie ou son œuvre dans toute bonne bibliothèque et dans l'internet.

4. Ceux qui comme Comenius croyaient à l'avènement prochain du royaume millénaire du Christ sont connus comme millénaristes ou chiliastes ( $\mathrm{du}$ grec $\chi \chi^{\prime} \lambda_{1} \alpha=$ mille). 
5. Comenius s'est appliqué à en créer une et présente les résultats de ses efforts dans le cinquième livre de la Consultation universelle, qui porte le titre Panglottia (Panglossie). Ce livre estresté aussi inachevé.

6. Comenius en compta plus de 2000 (2005: 63).

7. Comenius y consacre tout le chapitre XXI de la Methodus Linguarum Novissima (MLN) et le chapitre XXII de la Grande didactique.

8. Par plurilinguisme Comenius entend la maîtrise de plusieurs langues (MLN : XXI). Pour l'Union Européenne le multilinguisme désigne à la fois « la capacité d'une personne d'utiliser plusieurs langues et la coexistence de plusieurs communautés linguistiques dans une zone géographique donnée. [...] ce terme est utilisé pour décrire le nouveau domaine d'action de la Commission en faveur d'un climat qui soit propice à la pleine expression de toutes les langues et dans lequel l'enseignement et l'apprentissage de diverses langues peuvent s'épanouir " (Bruxelles, le 22.11.2005.COM (596) 2005 final. Communication de la Commission au Conseil, au Parlement européen, au Comité économique et social européen et au Comité des Régions. Un nouveau cadre stratégique pour le multilinguisme, p. 4).

9. Il y en a d'autres, par exemple, celle de Peter Laurenberg, professeur à Rostock et auteur de Pansophia sive Paedia philosophica (1633).

10. Comenius appelle les écoles «fabriques d'hommes» (ibid. $1992: 85$ ).

11. "L'homme naît apte à acquérir la connaissance des choses. D'abord parce qu'il est à l'image de Dieu [...]. Et puisque parmi toutes les qualités de Dieu domine l'omniscience, nécessairement l'image de cette qualité resplendit aussi chez l'homme [...] A notre esprit en revanche, on ne peut assigner de limite, ni dans le ciel, ni dans l'autre [vie], ni au-delà. Il s'élève aussi haut dans les cieux qu'il descend profondément dans l'abîme de l'abîme. Il pourrait pénétrer avec une vitesse incroyable dans des espaces mille fois plus étendus. Nierons-nous dès lors que tout lui soit accessible? Nierons-nous qu'il puisse tout contenir?» (Comenius $1992: 60-61$ ).

12. «... il n'est pas nécessaire de connaître complètement une langue. La besogne serait ridicule et inepte " (Comenius 1992 : 92). La seule langue qu'il faut essayer d'apprendre parfaitement est la langue maternelle.

13. "Certé quo plures quis Linguas tenuerit, eo major Menti confusion metuenda; quedmodum non ignota exempla, in hebes quid et stupidum desisse ingenia, polyglottiae studiis nimium distenta » (Comenius 1957 [1657] : 15/II ; 2005 : Ch. XXI : 9).

14. Lux in tenebris est un recueil des révélations de trois visionnaires assemblées, traduites en latin et publiées par Comenius. Elles prédisent le retour au trône de Bohême du roi Frédéric $\mathrm{V}$ (1596-1632, le repatriement prochain des émigrés réformés, la destruction de l'Antéchrist (du pape et des jésuites) et la chute des Habsbourg.

15. Opera didactica omnia (ODO) est le titre du recueil de 43 travaux pédagogiques de Comenius, écrits entre 1627 et 1657. Ils sont présentés en deux volumes folios, subdivisés en quatre sections. La première section du premier volume comprend onze textes rédigés entre 1627 et 1642, la deuxième huit textes écrits entre1642 et 1650. La première section du second volume contient 15 textes écrits entre 1650 et 1654 et la deuxième section neuf courts textes composés après l'arrivée de Comenius à Amsterdam, en 1656 et avant la publication des ODO en 1657.

16. Les piétistes représentent une importante secte luthérienne qui met l'accent sur la piété personnelle plutôt que sur le respect formel du dogme. Le principal représentant du piétisme était A.H. Francke (1663-1727), théologien et éminent pédagogue, fort influencé par Comenius.

17. Herder, Johann Gottfried (1744-1803), célèbre écrivain, philosophe et pédagogue allemand. Il est un des premiers à redécouvrir l'œuvre de Comenius et à souligner son importance.

18. Néo-humanisme, puissant mouvement intellectuel qui se développe en Allemagne à la fin du $18^{\mathrm{e}}$ et au début du $19^{\mathrm{e}}$ siècle. Les néo-humanistes mettent l'accent sur la langue et la civilisation grecque plutôt que sur le latin et la culture romaine. Les principaux représentants du 
mouvement néo-humaniste étaient Gesner, Heyne, Wolf, Herder, Wilhelm von Humboldt, etc. (Caravolas $2000: 126-130)$.

\section{RÉSUMÉS}

Combien de langues un homme devrait-il connaître : une ? deux ? plusieurs ? toutes ? comment les apprendre et comment les enseigner? Vieilles questions, toujours sans réponse.

À ma connaissance le premier à s'occuper de multilinguisme de manière approfondie, à proposer des solutions pertinentes et à esquisser une base théorique pour la didactique du multilinguisme est Jan Amos Comenius. Il le fait dans Didactica Magna (1657), dans Methodus linguarum Novissima (1649) et dans Panglottia, cinquième livre de son magnum opus De Rerum Humanarum Emedatione Consultatio Catholica (1966).

Dans mon exposé, j'examine l'opinion de Comenius sur les avantages et les inconvénients de l'unilinguisme (monoglottia), du bilinguisme (diglottia), du multilinguisme (polyglottia) et de la panglottie. Je me penche également sur son projet d'une éducation universelle pansophique et multilingue et sur les principes théoriques de base de sa didactique du plurilinguisme.

A la fin, j'explique les raisons pour lesquelles ses suggestions furent après sa mort oubliées, comment elles sont présentement redécouvertes par les spécialistes de la pédagogie des langues et pourquoi elles sont aujourd'hui adoptées et promues par l'UNESCO et l'Union Européenne.

How many languages should a man know : one? two? several? all? For what reason? How to learn them and how to teach them ? Old questions, still debated. To my knowledge, Jan Amos Comenius was the first to deal in detail with multilingualism, to propose pertinent solutions and to devise a theoretical basis for the didactics of multilingualism. He did so in Didactica Magna (1657), Methodus linguarum Novissima (1649) and in Panglottia, the fifth book of his magnum opus De Rerum Humanarum Emedatione Consultatio Catholica (1966).

In my presentation I examine Comenius' opinion on the advantages and inconveniences of monolingualism (monoglottia), bilingualism (diglottia), multilinguism (polyglottia) and panglottism; I also discuss his project for a universal, pansophical and multilingual education and the basic theoretical principles of his multilingualism didactics.

Finally, I explain the reasons for which his suggestions were forgotten after his death, how they are rediscovered today by language teaching specialists and why they are adopted and promoted now by UNESCO and the European Union.

\section{INDEX}

Keywords : unilinguisme, multilingualism, language teaching, Comenius, pansophism, universal education

Mots-clés : unilinguisme, multilinguisme, didactique, Comenius, pansophie, éducation universelle 


\section{AUTEUR}

JEAN ANTOINE CARAVOLAS

Université du Québec à Montréal 